\title{
Analysis of the correlation between micro-mechanical fields and fatigue crack propagation path in nodular cast iron
}

\author{
Andriollo, Tito; Zhang, Yubin; Fæster, Søren; Kouznetsova, Varvara
}

Published in:

Acta Materialia

Link to article, DOI:

10.1016/j.actamat.2020.02.026

Publication date:

2020

Document Version

Peer reviewed version

Link back to DTU Orbit

Citation (APA):

Andriollo, T., Zhang, Y., Fæster, S., \& Kouznetsova, V. (2020). Analysis of the correlation between micromechanical fields and fatigue crack propagation path in nodular cast iron. Acta Materialia, 188, $302-314$. https://doi.org/10.1016/j.actamat.2020.02.026

\section{General rights}

Copyright and moral rights for the publications made accessible in the public portal are retained by the authors and/or other copyright owners and it is a condition of accessing publications that users recognise and abide by the legal requirements associated with these rights.

- Users may download and print one copy of any publication from the public portal for the purpose of private study or research.

- You may not further distribute the material or use it for any profit-making activity or commercial gain

- You may freely distribute the URL identifying the publication in the public portal 


\title{
Analysis of the correlation between micro-mechanical fields and fatigue crack propagation path in nodular cast iron
}

Tito Andriollo $^{\mathrm{a}, \mathrm{d}}$, Yubin Zhang ${ }^{\mathrm{a}}$, Søren Fæster ${ }^{\mathrm{b}}$, Varvara Kouznetsova ${ }^{\mathrm{c}}$

${ }^{a}$ Department of Mechanical Engineering, Technical University of Denmark, Denmark

${ }^{\mathrm{b}}$ Department of Wind Energy, Technical University of Denmark, Denmark

c Department of Mechanical Engineering, Eindhoven University of Technology, The Netherlands

${ }^{\mathrm{d}}$ Corresponding author. Full address: Technical University of Denmark, Produktionstorvet, Building 425, room 225, 2800 Kgs. Lyngby, Denmark. E-mail: titoan@mek.dtu.dk

\begin{abstract}
The morphology of the fatigue crack in nodular cast iron has great influence on the material performance under cyclic loading. In contrast to previous phenomenological studies, the present work attempts to unravel a relation between the crack propagation path and the micromechanical fields developing at the microstructural level. To this end, a fatigue crack in a compact tension specimen is imaged with X-ray tomography and a new segmentation procedure based on digital volume correlation is used to distinguish the part of the crack growing in the matrix from the graphite nodules. The micromechanical fields surrounding the tip of the notch as well as the tip of the fully developed fatigue crack are estimated via 3D microstructure-resolved finite element models, whose size is maintained small compared to the specimen by using boundary conditions measured in-situ with digital image
\end{abstract}


correlation. The numerical fields are compared to the location of the crack nucleation site as well as to the crack growth direction. A weak correlation is found between the nucleation site and the equivalent plastic strain, suggesting that nucleation is controlled by plasticity occurring at a lower scale. On the other hand, a clear correlation is revealed between the crack growth direction and the direction perpendicular to the maximum principal stress. Based on this result, a mechanism is proposed to explain the step-like features characterizing the shape of the fatigue crack in nodular cast iron.

\section{Keywords}

Fatigue; Crack propagation; Cast iron; Digital volume correlation; Micromechanical modeling

\section{Introduction}

Modern nodular cast iron, also known as ductile iron or spheroidal graphite iron (SGI), is a structural material widely used in key industrial sectors like transport and energy production, due to its unique combination of castability, good mechanical properties and low price compared to traditional low-carbon steels [1]. Microstructurally, SGI consists of graphite particles - called nodules - embedded in a metal matrix, which can be either ferrite, pearlite or a mixture of the two depending on chemistry and processing route. It is well known that shape, size and distribution of the nodules can have significant influence on the SGI fatigue behavior, in terms of both crack initiation and propagation [2]. Recently, this aspect has received growing attention among researchers, mainly due to the fact that as castings grow 
bigger and heavier, the microstructural features become more difficult to control during manufacturing and the formation of graphite morphologies that deviate markedly from the ideal nodular shape becomes common [3-5].

One way to explain the effect of the nodules geometrical and topological parameters on the fatigue behavior of SGI is, from a mechanistic standpoint, via the concept of roughness induced crack closure. As originally discussed in [6], coarser microstructures result in more pronounced deflections of the crack path, leading to a rougher fracture surface and to enhanced crack closure. Recent studies confirm the dominance of this closure mechanism in SGI and its tight connection with the nodules shape [7,8]. Another explanation, proposed in [9], assumes that the nodules act as crack arresters and their effectiveness in blunting the crack tip is proportional to the nodules average size and shape factor. Despite focusing on different mechanisms, it is evident that both explanations acknowledge the fundamental effect played by the nodules in determining the crack propagation path, considered in terms of both tortuosity in the matrix as well as number, size and shape of the nodules intercepted by the crack.

The above argumentation leads to the conclusion that understanding the factors controlling the crack propagation path is crucial. In this respect, experimental evidence shows that the fatigue crack in SGI grows transgranularly by linking the nodules $[10,11]$. These appear to act as crack attractors [12], leading to a higher graphite fraction on the fracture surface than in the bulk of the material [13]. Also, a crack might or might not propagate depending on the presence of clusters of nodules near its tip [14], which seem to be associated with load shielding effects [15]. These facts suggest that an analogy exists with particle-reinforced 
metal-matrix composites, where it has been clarified that the crack growth, including the occurrence of shielding and anti-shielding phenomena, is driven by the particle-induced microstructural stress field [16,17]. Similar conclusions hold regarding fatigue crack propagation in cast $\mathrm{Al}$ alloys with respect to the impact of Si-particles and Fe-rich intermetallics $[18,19]$. Consequently, it can be hypothesized that the crack path in SGI is also primarily determined by the local micromechanical field variations caused by the presence of the nodules.

In order to confirm this hypothesis, measurements of the stress and/or strain fields near the nodules surrounding the crack tip would be necessary. Concerning this, focused ion beam milling and digital image correlation were applied by Kasvayee et al. [20] to quantify the 2D microstructural strain distribution on the surface of a compact tension sample ahead of microcracks formed during cyclic loading. However, a qualitative link between the deformation pattern and the observed crack propagation direction could not be established, which, according to the authors, could be a consequence of the artifacts associated with 2D measurements. On the other hand, experimental characterization of the full 3D deformation field at the level of the microstructure is very challenging. The reason is that the limited number of matrix grains between neighboring nodules precludes the application of energy dispersive X-ray diffraction [21], whereas measurements using X-ray Laue micro-diffraction $[22,23]$ require long time to probe a volume sufficiently large to be statistically relevant and this may lead to stress relaxation during the measurement. In addition, absorption tomography combined with digital volume correlation, which have proved to be very 
effective for the determination of the local stress intensity factor in SGI [24-26], lacks spatial resolution if the nodules alone are used as markers.

In the present work, in order to overcome the difficulties associated with direct experimental measurements, the micromechanical fields surrounding the tip of the notch as well as the tip of the fully developed fatigue crack in a compact tension specimen are estimated numerically. This is achieved by means of high-fidelity finite element models of the microstructure, whose size is maintained small compared to that of the specimen by using boundary conditions measured in-situ with digital image correlation. The computed micromechanical fields are compared to the location of the crack nucleation site as well as to the crack growth direction. The outcome of the analysis is used to formulate a hypothesis for the mechanism of formation of the step-like features characterizing the fatigue crack in SGI.

\section{Experimental}

\subsection{Material}

The material used in the present investigation is SGI extracted from a block with size of 900mm x 900mm x 250mm of casting grade EN-GJS400-18LT. The microstructure consists of graphite nodules of mean diameter $\approx 80 \mu \mathrm{m}$, with graphite volume fraction $13.9 \%$. The nodules are embedded in the predominantly ferrite matrix, with $\approx 1 \%$ pearlite. Young's modulus, yield stress $(0.2 \%$ proof stress $)$ and tensile strength of the considered SGI were determined from the uniaxial tensile stress-strain curves reported in Figure 1 (a) and are equal to $172 \mathrm{GPa}, 220 \mathrm{MPa}$ and $354 \mathrm{MPa}$, respectively. The cyclic hardening behavior was assessed from the hysteresis loops shown in Figure 1 (b), obtained by subjecting a cylindrical 
specimen of diameter $10 \mathrm{~mm}$ and gauge length $30 \mathrm{~mm}$ to 10 stress cycles between $-250 \mathrm{MPa}$ and +250 MPa. An elastoplastic model describing the cyclic behavior of the present SGI will be presented in Section 3.2.

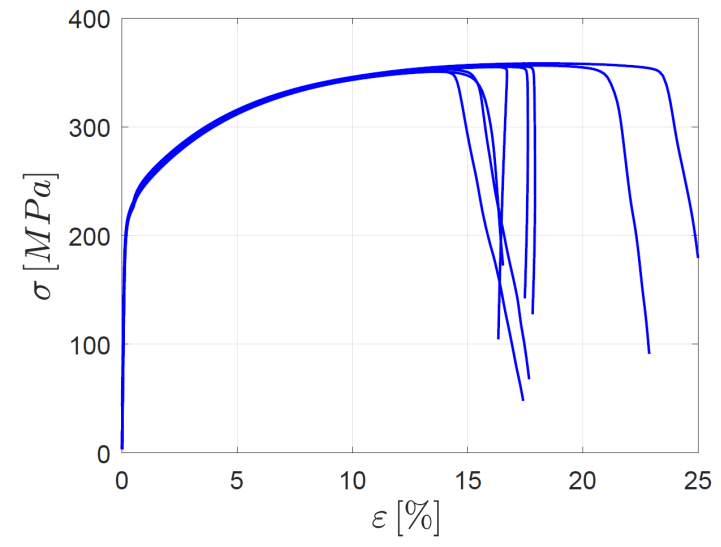

(a)

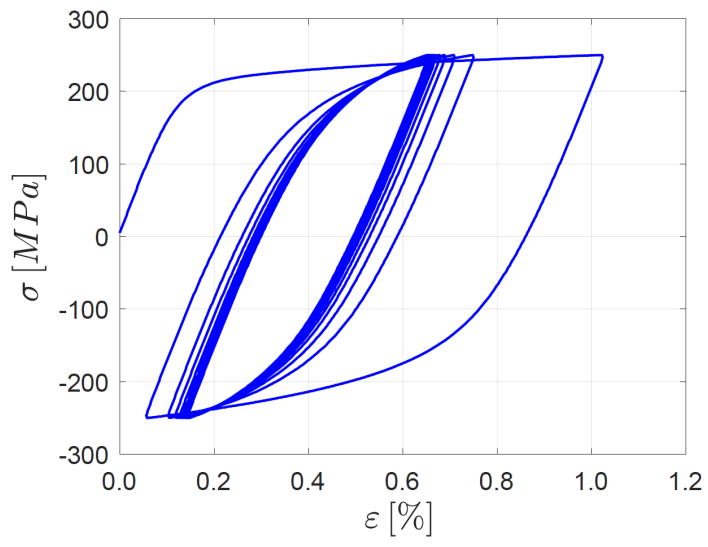

(b)

Figure 1 - Uniaxial mechanical testing of the SGI used in the present work. (a) Monotonic tensile curves (b) Hysteresis loops obtained by applying 10 loading cycles between $-250 \mathrm{MPa}$ and $+250 \mathrm{MPa}$.

\subsection{Crack growth test with optical \& X-ray imaging}

A compact tension specimen with the in-plane dimensions indicated in Figure 2 (a) and thickness $1.5 \mathrm{~mm}$ - which fulfill the requirements of the ASTM standard E647 [27] - was manufactured via electro-discharge machining. Both faces of the specimen were mirrorpolished. Subsequently a white and a black speckle pattern was applied on one face of the specimen with a precision airbrush, resulting in a speckle size of approx. $100 \mu \mathrm{m}$.

The specimen was subjected to the testing procedure summarized in Figure 2 (b). First, a Zeiss Xradia 520 Versa Computed Tomography (CT) system was used to obtain an initial 3D scan (\#1) of the microstructure. The X-ray scanner was equipped with a tungsten target 
and an acceleration voltage of $140 \mathrm{kV}$ was applied to generate $\mathrm{X}$-rays with energies up to $140 \mathrm{keV} .3801$ projections were acquired during a full $360^{\circ}$ rotation of the specimen. The detector size was $2 \mathrm{k} \times 2 \mathrm{k}$ and projection images with a binning of 1 were acquired to obtain the highest possible resolution. A Feldkamp reconstruction algorithm [28] for cone beam reconstructions was applied resulting in a reconstructed 3D scan with a voxel size of 1.904 $\mu \mathrm{m}$. Re-sampling was employed to align the scan to the $\mathrm{X}-\mathrm{Y}-\mathrm{Z}$ coordinate system of the specimen visualized in Figure 2 (a). The specimen was then mounted on a $3 \mathrm{kN}$ electropulse testing machine and subjected to a sinusoidal load with frequency $5 \mathrm{~Hz}$, peak load $650 \mathrm{~N}$ and amplitude $292.5 \mathrm{~N}$, corresponding to a fatigue ratio of 0.1 and an initial $\Delta K_{I}$ of 15.5 $\mathrm{MPa} \cdot \mathrm{m}^{1 / 2}$. During loading, 2D images of the painted face of the specimen were acquired at regular interval at both maximum and minimum load by means of an Imager E-lite $2 \mathrm{M}$ digital camera with 1600x1200 pixels ${ }^{2}$, which allowed for a pixel size of $8 \mu \mathrm{m}$. After 40000 cycles, which sufficed to grow a fully developed crack of $\approx 2.2 \mathrm{~mm}$, the loading was discontinued and the specimen was moved into the CT system for a new 3D scan (\#2). To improve the visualization of the crack, a small wedge was inserted in the notch and used to keep the crack open during the CT scanning operation. Finally, the specimen was reloaded with the same loading parameters for additional 10000 cycles, resulting in additional crack growth at 4.0 $\mathrm{mm}$, after which it was removed from the testing machine and subjected to one more CT scan (\#3), in order to determine the crack propagation direction upon reloading. 

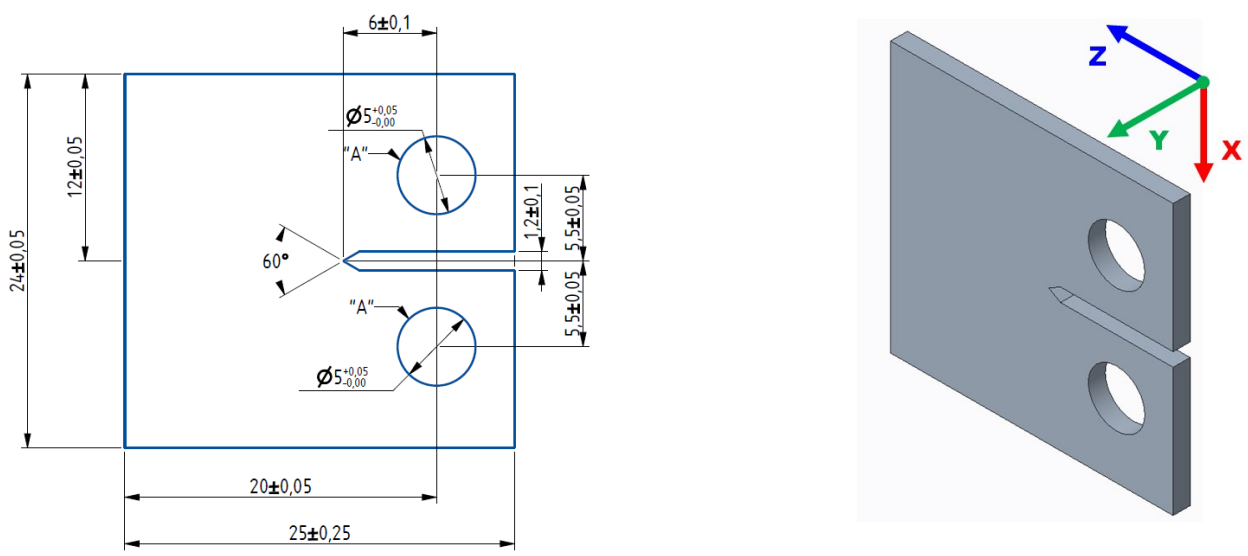

(a)

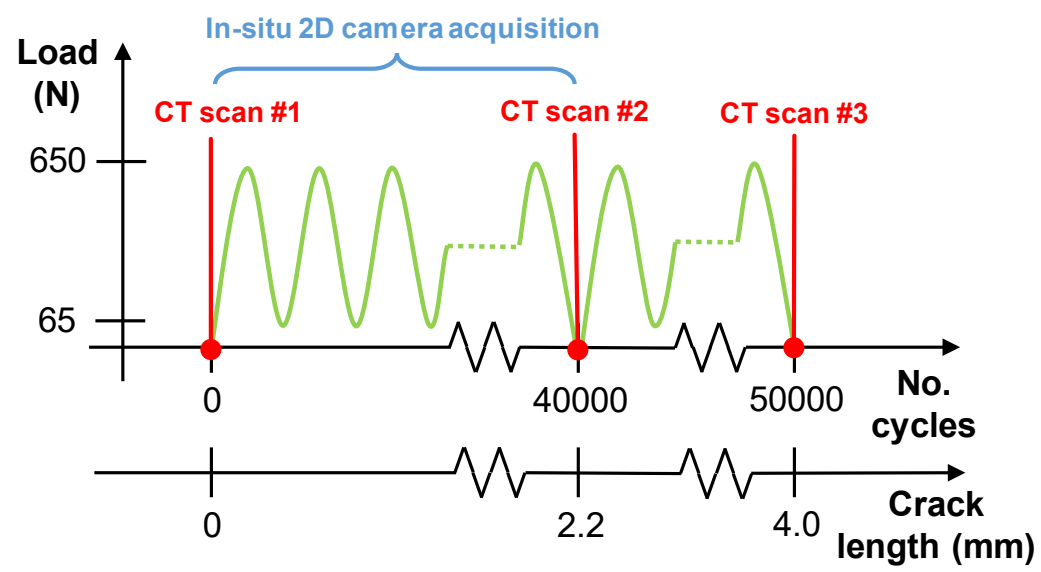

(b)

Figure 2 - Crack growth test combined with digital camera and X-ray imaging. (a) Geometry of the compact tension specimen, with dimensions given in $\mathrm{mm}$. (b) Testing procedure.

\subsection{Crack surface reconstruction}

The 3D shape of the fatigue crack was reconstructed from the CT scans by means of a dedicated reconstruction procedure that allows distinguishing between the crack and the nodules. The procedure is inspired by that of Limodin et al. [24] and is based on the correlation residual of the global digital volume correlation (G-DVC). 
As described in detail elsewhere, e.g. [29], G-DVC allows finding a mapping between a reference and a deformed 3D image by decomposing the displacement field into a weighted sum of shape functions, similar to standard finite element formulations. Under the assumption of continuous shape functions, the deformed image cannot be perfectly matched to the reference image if the real displacement field contains discontinuities. Therefore, the correlation residual defined as the difference between the deformed image - corrected for by the continuous G-DVC displacement - and the reference image highlights regions where material discontinuities have occurred, meaning that it can effectively indicate a crack path. However, in SGI high values of the correlation residual can also be associated with the debonding of the graphite particles from the matrix. To identify the crack unambiguously, two strategies have been suggested in the literature. The first one consists of describing the crack surface via dedicated shape functions and choosing the weighting coefficients so that the correlation residual over the crack is maximized [30], whereas the second one is based on segmenting the correlation residual via region-growing algorithms [24]. As discussed by Rannou et al. [30] both have limitations when it comes to accuracy. Indeed, difficulties arise with the first method in detecting the crack tip and in capturing overhangs or non-smooth transitions, whereas region-growing algorithms can lead to an excessively rough crack surface and presence of holes.

An alternative strategy is introduced in this investigation to achieve an accuracy high enough to enable a high-fidelity representation of the crack in the matrix region between neighboring nodules, which is crucial in the present context. Specifically, the first step is standard and consisted in running G-DVC with the software Avizo ${ }^{\circledR}$ [31] using CT scan \#1 as the 
reference image and $\mathrm{CT}$ scan $\# 2$ as the deformed image to compute the correlation residual. As expected, this identifies the crack but also some matrix-nodules debonding, as Figure 3 (a) and Figure 3 (b) reveal. Next, the correlation residual and the reference image were both segmented via simple thresholding and combined via a binary OR operation. Subsequently, a moderate morphological dilation was applied followed by removal of all the connected components except the largest. As Figure 3 (c) shows, the outcome of this segmentation process is a binary image that identifies the crack together with all the intersected nodules. At this point, the morphological skeleton was computed for each $\mathrm{X}-\mathrm{Z}$ slice - refer to the coordinate system of Figure 2 - of the segmented image. It is worth mentioning that, for a $2 \mathrm{D}$ binary image, the skeleton is defined as the locus of the ridges of its medial axis transform [32] which, in plain words, correspond to the ridges of the 3D plot representing the distance to the boundaries of the objects in the image. Finally, for each X-Z slice, the secondary skeleton branches were trimmed and the part of the skeleton overlapping the nodules was removed based on the original segmentation of the reference image. The result, visualized in Figure 3 (d), consists of a one-pixel-thick surface representing the part of the crack located in the matrix. Comparison with the CT scan $\# 2$ showed that the deviations from the real crack geometry are expected to be less than $10 \mu \mathrm{m}$.

The entire reconstruction procedure was repeated twice. The first time, as already mentioned, the CT scan \#1 was used as the reference image and the CT scan \#2 as the deformed image in order to reconstruct the full crack morphology after 40000 cycles. The second time, the CT scan \#3 was used as the deformed image while keeping the CT scan \#1 as the reference image and the procedure was applied to a limited region only in order to determine the crack 
propagation direction upon reloading. Further details of the computation of the $3 \mathrm{D}$ crack propagation direction are reported in the supplementary material.

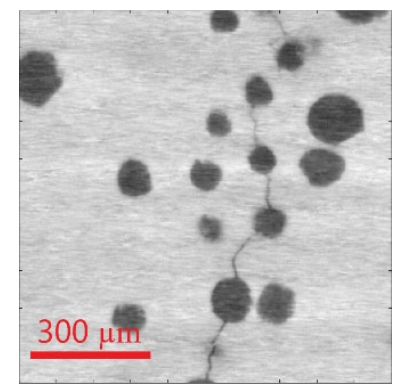

(a)

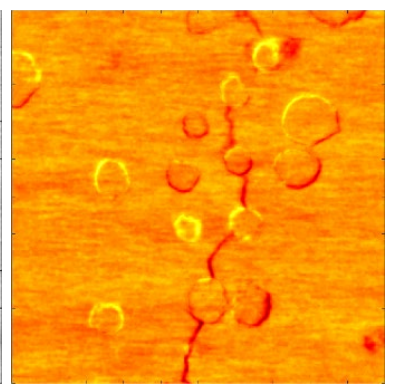

(b)

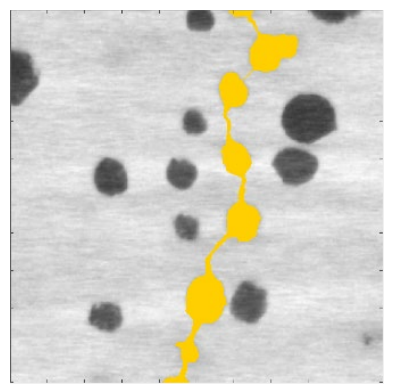

(c)

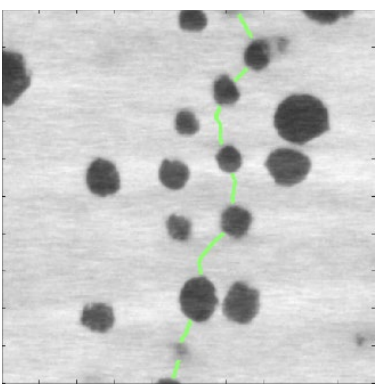

(d)

Figure 3 - Crack surface reconstruction procedure for a cropped X-Z slice of size $950 \times 950 \mu \mathrm{m}^{2}$. (a) Image after deformation containing the crack. (b) Correlation residual of G-DVC. (c) Binary image resulting from the segmentation process (yellow $=1$, transparent $=0$ ), superimposed onto the reference image, i.e. before deformation. (d) Processed skeleton of the segmented binary image representing the part of the crack propagating in the matrix, superimposed onto the reference image. Note that the skeleton thickness has been increased for visualization purposes.

\section{Finite element modelling}

Two finite element models were developed in order to estimate the micro-mechanical fields arising in the microstructure of the compact tension specimen. The first one corresponds to the volume surrounding the tip of the notch at the beginning of the cyclic loading, whereas the second one represents the volume surrounding the tip of the fully developed crack after 40000 cycles. Both models consist of an inner region where the microstructure - matrix plus graphite particles - is resolved and a homogenized outer region which represents the effective behavior of SGI. The following three sections describe the geometry of the two models, the 
constitutive equations used to simulate the behavior of the microstructural constituents and the boundary conditions applied during the simulations.

\subsection{Geometry}

Each model was generated by extracting from CT scan \#1 a sub-volume having the shape of a square prism with faces aligned with the coordinate axes. The height of the sub-volume was $1.5 \mathrm{~mm}$, corresponding to the thickness of the specimen, whereas the size of its base was $1.6 \times 1.6 \mathrm{~mm}^{2}$. Depending on the model type, the sub-volume was centered either on the notch tip or on the crack front after 40000 cycles. Note that the location of the latter - not available in CT scan \#1 - was retrieved with the procedure described in Section 2.3.

The sub-volume was filtered and segmented to separate the nodules from the matrix. In doing so, particles with equivalent diameter smaller than $15 \mu \mathrm{m}$ were discarded, as they are likely to represent the small porosities and inclusions which are commonly found in the SGI matrix [33]. Nodules whose centroid was located outside the inner region of the sub-volume were discarded as well. This inner region, which corresponds to the microstructure-resolved region of the model as mentioned previously, was defined as the volume generated by a circle perpendicular to the $\mathrm{Y}$-axis and with radius $400 \mu \mathrm{m}$, whose center was moved along the points located $115 \mu \mathrm{m}$ ahead of either the notch tip or the crack tip, depending on the model type. A 2D view of the boundary of the inner region (marked in red) together with the outcome of the segmentation procedure for the crack tip model (green $=$ nodules inside the inner region, yellow $=$ nodules outside, blue $=$ matrix $)$ is shown in Figure 4 , whereas a 3D slice-by-slice view is provided in Video 1 in the supplementary data. 
The abovementioned procedure led to a $3 \mathrm{D}$ binary image having the value 1 at the locations occupied by the nodules and the value 0 elsewhere. This was used to create a signed distance map, which was then passed on to a dedicated meshing toolbox [34]. While generating the mesh, a target minimum element side-length of $5 \mu \mathrm{m}$ was imposed, which was deemed sufficient in the light of the considerations reported in Appendix A. It is worth remarking that the total graphite volume fraction can be affected by the meshing process, as this involves a certain degree of particle smoothing. To avoid this effect, the particles contained in the 3D binary image used as the input were expanded/contracted isotropically about their centroids by an amount determined iteratively such that the total graphite volume fraction was preserved in the final mesh. After meshing, the elements located inside the sub-volume inner region and not associated with the nodules were assigned to the matrix. The remaining elements not associated with the nodules were assumed to form the homogenized outer region of the model. The final meshes, each containing $\approx 6.5$ million 4-node hybrid displacementpressure elements, are shown in Figure 5.

One last aspect relates to the implementation of the notch and of the crack in the respective models. For reasons related to the technical features of the meshing toolbox used in this study, the notch was meshed explicitly and subsequently assigned negligible stiffness (1 MPa). Concerning the crack, it was inserted in the mesh of the corresponding model by duplicating the nodes along the element facets that best fitted the crack geometry. The details of this operation are reported in the supplementary data related to this article. Note that the crack was assumed stationary in the model, i.e. crack growth was not simulated. 

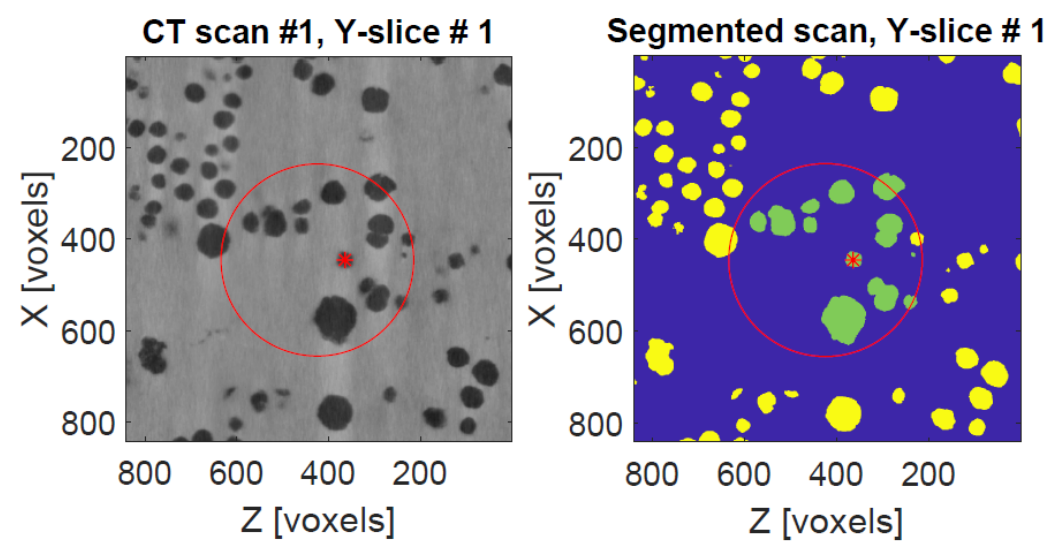

Figure 4 - Segmentation procedure for the crack tip model. On the left-hand side, an X-Z slice of the subvolume extracted from the CT scan \#1 is shown, where the red circle represents the boundary of the inner region and the red star indicates the crack tip. On the right-hand side, the same slice after segmentation is depicted, where the green color indicates the nodules inside the inner region and the yellow the nodules outside the inner region. See Video 1 in the supplementary data for a slice-by-slice 3D visualization.

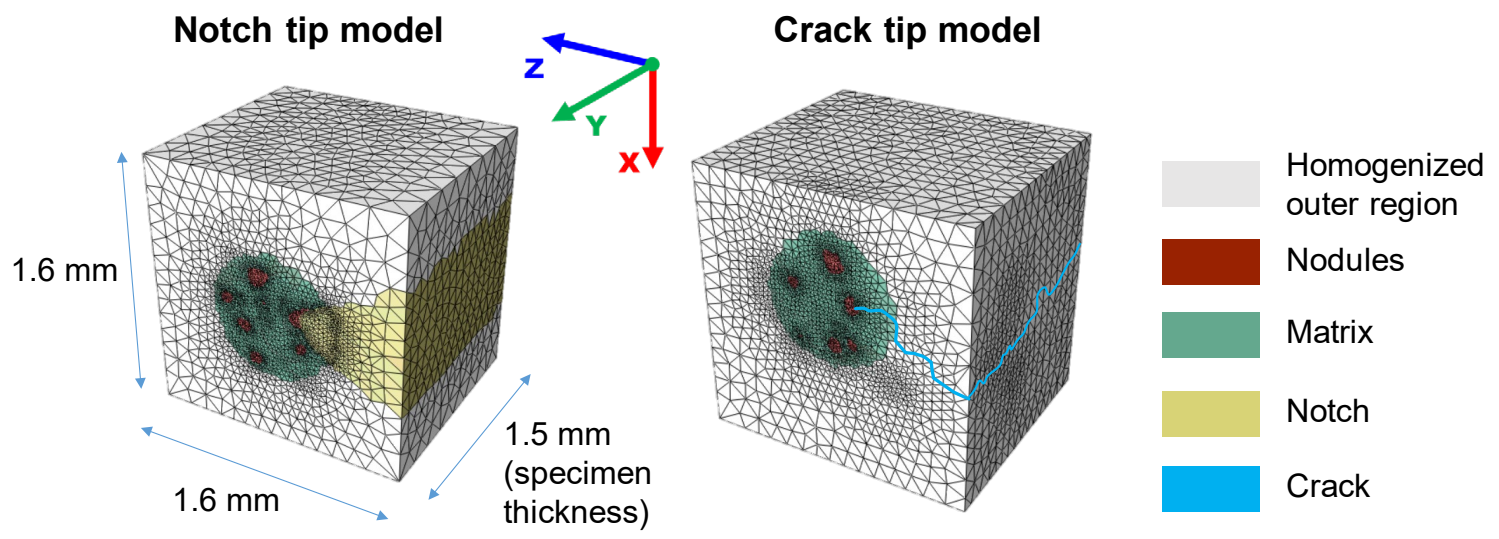

Figure 5 - The two finite element models used in the present investigation.

\subsection{Constituent material models}

The graphite nodules were assumed to behave linearly elastically. Their highly inhomogeneous and anisotropic internal structure was accounted for by means of the approach described in detail in [35]. The overall idea is that each nodule is divided into an 
external shell and a core. While the former is assumed isotropic, different orientations of the elastic symmetry and anisotropic moduli are assigned to each mesh element in the core based on the element location with respect to the centroid of the nodule. This allows for an approximate representation of the strong gradient in mechanical properties observed inside the nodules [36,37]. It is worth remarking that the method is not accurate for nodules that merged at some point during solidification to form aggregates. In such (limited) cases, the aggregate was first separated into individual particles by means of a method based on a watershed algorithm, whose details can be found in the supplementary data related to this article. The values of the transversely isotropic elastic moduli used for the core and of the isotropic elastic moduli used for the external shell of the nodules are listed in Table 1, whereas the corresponding values of the thermal expansion coefficients as functions of temperature can be found in the supplementary data. A thorough explanation for the choice of these parameters can be found in [35].

The interface between the nodules and the matrix was modelled using the surface-based cohesive formulation available in ABAQUS [38], which was the finite element software used in the present investigation. The traction-separation law was assumed linear elastic with constant normal stiffness $10 \mathrm{MPa} / \mu \mathrm{m}$ and shear stiffness $5 \mathrm{MPa} / \mu \mathrm{m}$. As discussed in [35], this assumption was introduced to represent the weak bonding between the nodules and the matrix reported in the literature [12].

Concerning the matrix, it is worth remarking that the mean size of the grains surrounding a graphite nodule is expected to roughly equal to half of the nodule equivalent diameter [22]. Therefore, grain-related effects may have a significant impact on the micromechanical fields 
developing in between the nodules. However, the non-destructive characterization of the 3D matrix grain structure would require advanced techniques that were not available in the present investigation. Consequently, it was chosen to model the matrix as an isotropic, homogeneous medium. This assumption is widely adopted in the SGI literature [10] and is usually justified by the random texture of the ferrite grains. In the present context, the validity of this assumption will be assessed in the next sections based on the ability of the considered finite element models to predict the fatigue crack nucleation site and growth direction. Following Rabold and Kuna [39], the matrix constitutive behavior was described via a rateindependent small-strain elastoplastic model, which, for the sake of simplicity, was assumed to apply to the homogenized region as well, although with different parameters. The model is based on a von Mises-type yield function:

$$
\Phi=\sqrt{3 J_{2}(\boldsymbol{\sigma}-\boldsymbol{\alpha})}-\sigma_{y}\left(\varepsilon_{e q}^{p l}\right) \leq 0
$$

where $J_{2}(\boldsymbol{\sigma}-\boldsymbol{\alpha})$ is the $2^{\text {nd }}$ deviatoric invariant of the difference between the Cauchy stress $\boldsymbol{\sigma}$ and the back-stress $\boldsymbol{\alpha}$ and $\varepsilon_{e q}^{p l}$ is the equivalent plastic strain. The function $\sigma_{y}\left(\varepsilon_{e q}^{p l}\right)$ describes the isotropic part of the hardening behavior and it is assumed to have the following expression:

$$
\sigma_{y}=Q_{0}+Q_{\infty}\left(1-e^{-b \varepsilon_{e q}^{p l}}\right)
$$

with $Q_{0}, Q_{\infty}$ and $b$ as material parameters. The standard additive decomposition of the strain into an elastic, thermal and plastic part is considered, where the elastic strain is related to the Cauchy stress via Hooke's law. The evolution of the plastic strain $\boldsymbol{\varepsilon}_{\mathbf{p}}$ is dictated by the flow rule: 


$$
\dot{\boldsymbol{\varepsilon}}_{\mathbf{p}}=\dot{\lambda} \frac{\partial \Phi}{\partial \boldsymbol{\sigma}}=\dot{\lambda} \frac{3}{2} \frac{(\mathbf{s}-\boldsymbol{\alpha})}{\sigma_{y}}
$$

where $\boldsymbol{s}$ is the deviatoric part of the Cauchy stress, $\lambda$ is the plastic multiplier and the operator ( ) denotes an infinitesimal increment. The evolution equation of the back-stress $\boldsymbol{\alpha}$ is of Armstrong-Frederick type:

$$
\dot{\boldsymbol{\alpha}}=\dot{\lambda} H \frac{(\mathbf{s}-\boldsymbol{\alpha})}{\sigma_{y}}-\dot{\lambda} \gamma \boldsymbol{\alpha}
$$

where $H$ and $\gamma$ are kinematic hardening parameters. The values of the model parameters, listed in Table 2, were determined as follows.

For the matrix, the values of the Young's modulus $E$, Poisson's ratio $v$ and thermal expansion coefficient $\alpha$ were taken from a previous work [40]. The plastic parameters $Q_{0}$, $Q_{\infty}, b, H$ and $\gamma$ were obtained following the framework of the first-order computational homogenization [41] by constructing a representative volume element (RVE) of the considered SGI microstructure and fitting its macroscopic response - computed via finite element simulations - to the cyclic data of Figure 1 (b). First, the values of $H$ and $\gamma$ were determined by fitting the data limited to the last cycle, assuming that negligible isotropic hardening takes place within that cycle. Then, the values of $Q_{0}, Q_{\infty}$ and $b$ were estimated by fitting the full cyclic data. The details of this data-fitting procedure involving RVE-based finite element simulations are reported in Appendix A.

For the homogenized region, which represents the effective behavior of SGI at the macroscale, the value of the Young's modulus was taken from the measurement data presented in Section 2.1. Standard literature values were considered for the Poisson's ratio and the thermal expansion coefficient. The values of the plastic parameters $Q_{0}, Q_{\infty}, b, H$ and $\gamma$ were 
determined by fitting the response of a single-element finite element model to the cyclic data of Figure 1 (b). As was done for the matrix, the values of $H$ and $\gamma$ were found first by fitting the data limited to the last cycle. After that, the values of $Q_{0}, Q_{\infty}$ and $b$ were estimated by fitting the full cyclic data.

Table 1 - Assumed elastic moduli of the graphite forming the core and the external shell of the nodules [40]. Values are in GPa, except for the dimensionless Poisson's ratio $v$.

\begin{tabular}{ccccc|ccc}
\hline & \multicolumn{3}{c}{ Core } & \multicolumn{2}{c}{ External shell } \\
\hline$C_{11}$ & $C_{44}$ & $C_{12}$ & $C_{13}$ & $C_{33}$ & $E$ & $v$ \\
\hline 166 & 0.66 & 26 & -0.38 & 4.4 & 10 & 0.2 \\
\hline
\end{tabular}

Table 2 - Parameters of the elastoplastic model describing the behavior of the matrix and of the homogenized SGI.

\begin{tabular}{ccccccccc}
\hline & $E[\mathrm{GPa}]$ & $v$ & $\alpha\left[{ }^{\circ} \mathrm{C}^{-1}\right]$ & $Q_{0}[\mathrm{MPa}]$ & $Q_{\infty}[\mathrm{MPa}]$ & $b$ & $H[\mathrm{GPa}]$ & $\gamma$ \\
\hline Matrix & 205 & 0.29 & $1.35 \times 10^{-5}$ & 275 & 68 & 3.7 & 11 & 790 \\
SGI & 172 & 0.26 & $1.20 \times 10^{-5}$ & 138 & 115 & 9.2 & 78 & 725 \\
\hline
\end{tabular}

\subsection{Boundary conditions}

The finite element models were subjected to two loading steps. First, the processing step representing the final part of the solid-state cooling was simulated by prescribing a uniform temperature change from $500^{\circ} \mathrm{C}$ to $20^{\circ} \mathrm{C}$, while leaving the model boundaries unconstrained. This was done in order to include the residual stress state associated with the mismatch in thermal contraction between the nodules and the matrix [22] into the subsequent simulations. Next, both models were subjected to cyclic loading simulating the conditions experienced during the crack propagation test. To this end, the X- and Z-displacement fields over the painted face of the specimen were retrieved by applying subset-based digital image correlation (DIC) to the 2D digital images acquired in-situ during cyclic loading, see Section 
2.2. Specifically, the displacements at both maximum and minimum load at the beginning of the test (resp. after 40000 cycles) were retrieved from images taken after 100 (resp. 40000) cycles and used to prescribe the boundary conditions in the notch (resp. crack) tip model. The DIC was run using a subset size of 65 pixels and a step size of 2 pixels, which produced results affected by an error - estimated by comparing two images of the unloaded, uncracked specimen - below $0.1 \mu \mathrm{m}$. Figure 6 shows contours of the DIC displacement at maximum load at the beginning of the test and after 40000 cycles. The DIC output was used to prescribe the X-and Z-displacement on the boundaries of both the notch tip and the crack tip models perpendicular to the $\mathrm{X}-\mathrm{Z}$ plane, assuming that the same displacement occurred at the points having equal $\mathrm{X}$ - and Z-coordinates, but different $\mathrm{Y}$-coordinate. The displacement in the $\mathrm{Y}$ direction was left unconstrained, as no information was available in this regard. For each model, five consecutive loading cycles with identical boundary conditions (i.e. prescribed boundary displacement at maximum and minimum load) were simulated, in order to achieve stabilization of the micromechanical fields, i.e. hardening.

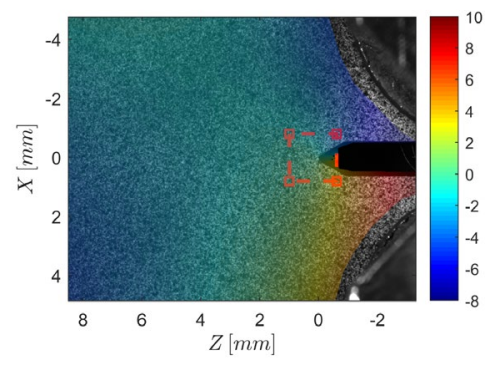

(a)

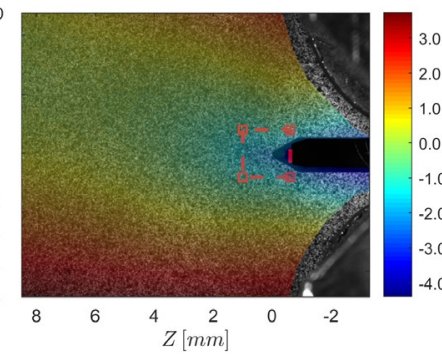

(b)

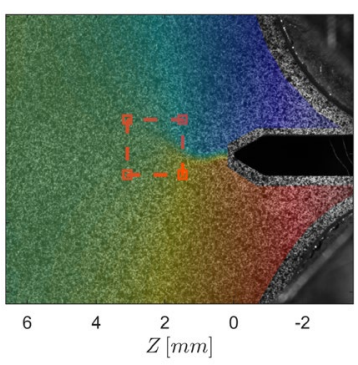

(c)

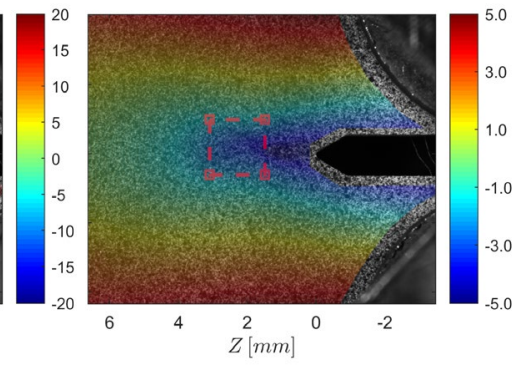

(d)

Figure 6 - DIC displacement at maximum load over the painted face of the compact tension specimen in $\mu \mathrm{m}$. (a) X-component at the beginning of the test. (b) Z-component at the beginning of the test. (c) X-component after 40000 cycles. (b) Z-component after 40000 cycles. The red square indicates the boundaries of the notch tip model in (a), (b) and the boundaries of the crack tip model in (c), (d). 
For comparison, the DIC data prescribed along the model boundaries was contrasted with the displacements obtained from a simple 2D finite element model of the entire compact tension specimen, assuming homogenized material behavior. The comparison can be found in Appendix B, together with the investigation of the sensitivity of the microstructural model response with respect to the boundary conditions.

\section{Results}

\subsection{Morphology of the crack}

Figure 7 (a) shows the morphology of the crack after 40000 cycles reconstructed with the procedure described in Section 2.3. To the authors' best knowledge, it is the first time that a fully developed fatigue crack in SGI is characterized in 3D with an accuracy that allows a clear distinction between the part of the crack growing in the matrix and the surrounding nodules. The data is valuable as simple visual inspection demonstrates that the crack deviations from the ideal $\mathrm{Y}-\mathrm{Z}$ propagation plane - proportional to the color change - are significantly larger than the average size of the nodules $(80 \mu \mathrm{m})$. This implies that the roughness of the crack may not be considered the result of a planar crack whose surface merely "adapts" locally to the shape of the individual nodules intercepted along the way. It can therefore be hypothesized that longer-range micro-mechanical fields play a role in driving the crack, as will be investigated in the following. 


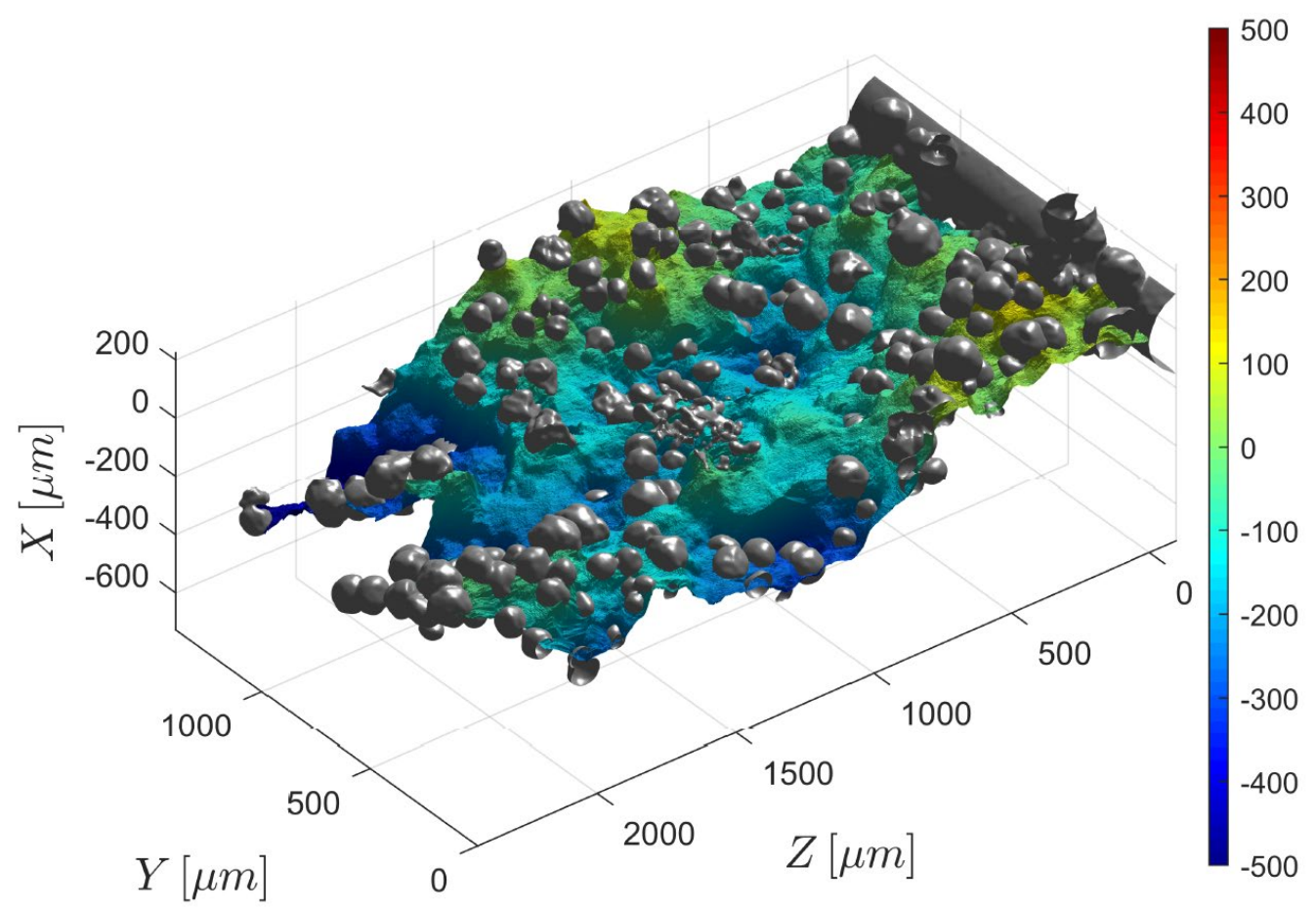

(a)

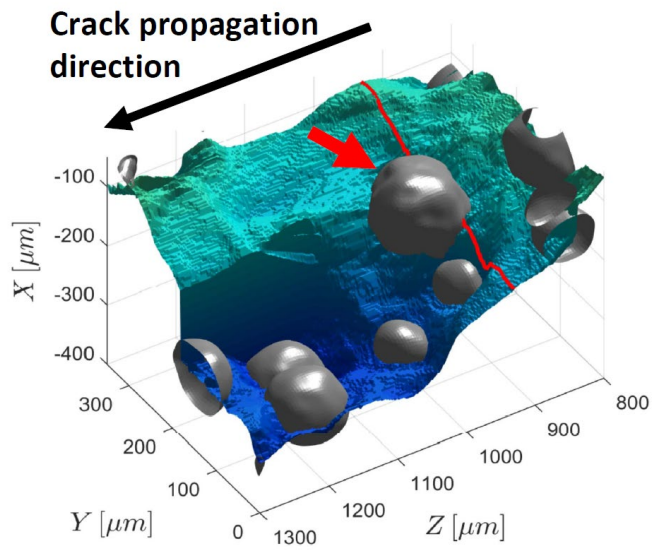

(b)

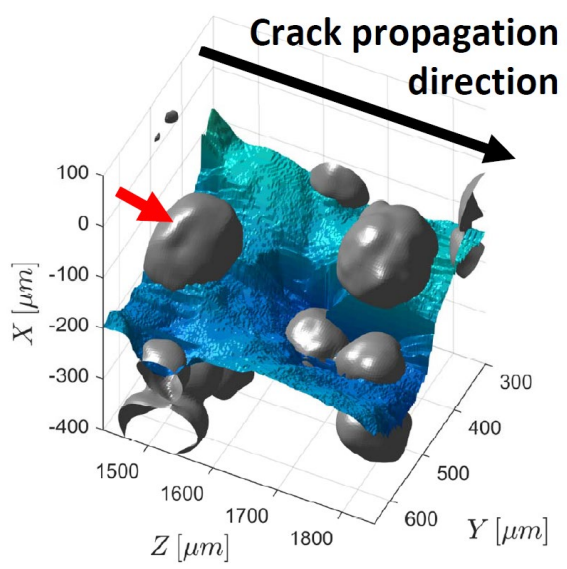

(c)

Figure 7 - (a) Reconstructed morphology of the crack after 40000 cycles. The color represents the crack height with respect to the ideal propagation plane in $\mu \mathrm{m}$. The nodules intersecting the crack and the notch tip (semi-cylindrical surface on the right-hand side) are visualized in gray. To simplify the figure generation process the part of the crack inside the nodules was retained, even though it is not trustworthy. (b), (c) Closeups showing the presence of steps with step wall parallel to the crack growth direction. 
An additional feature of the crack morphology is the existence of steps, characterized by a step wall parallel to the crack growth direction and wall height larger than the mean nodules size. Close-ups of two of these steps are provided in Figure 7 (b) and (c). The formation of such steps during fatigue loading seems to be a distinctive feature of SGI, as it was already reported in previous studies [25,42]. In particular, Limodin et al. [25] found a strong correlation between the formation of one such step and a delay in the local crack propagation rate, which was ascribed to the activation of type II and type III loading modes. However, the former authors could not provide any plausible reasons for the formation of these steps, whose nature is still unknown at present. A possible mechanism will be presented here in Section 5.

It is worth remarking that the present fatigue crack was grown under conditions that correspond to the mid Paris regime for the material considered [43]. For both ferritic and pearlitic SGIs, 2D microscopy observations indicate that the appearance of the crack surface is similar in the entire stable crack propagation regime [15], as well as at different fatigue ratios and maximum stress intensity factors [8]. Therefore, it is likely that some of the features of the crack identified in Figure 7 are characteristic of a rather wide range of stable crack propagation regimes.

\subsection{Correlation between micromechanical fields and crack nucleation site}

In order to identify possible correlations between the micromechanical fields and the crack nucleation site, the generic field $f$ predicted with the notch tip model and defined over the 
corresponding finite element mesh was projected onto a 3D grid with voxel size equal to that of the binary image describing the crack shape. Then, for every X-Z slice of the grid, the quantity $q_{c}=f_{P} / \bar{f}_{\text {circle }}-1$ was computed, where $f_{P}$ is the value of $f$ at the point $\mathrm{P}$ at which the crack nucleated and $\bar{f}_{\text {circle }}$ is the average value of $f$ over a circle of radius $\mathrm{R}_{\mathrm{c}}$ centered at P. It is remarked that only points (i.e. voxels) belonging to the matrix phase were taken into account. The values at maximum load of the following scalar fields were considered: maximum principal plastic strain $\varepsilon_{I, \text { max }}^{p l}$, maximum principal stress $\sigma_{I, \text { max }}$, von Mises stress $\sigma_{e q}$ and triaxiality $\eta$, defined as the ratio between the hydrostatic part of the stress and $\sigma_{e q}$. The equivalent plastic strain increment during one cycle $\Delta \varepsilon_{e q}^{p l}$ was considered as well. Figure 8 shows $q_{c}$ for the five scalar fields listed above. It can be seen that $q_{c}$ is close to zero for all stress-related quantities, meaning that the crack nucleated at points that did not have values of $\sigma_{I, \max }, \sigma_{e q}$ and $\eta$ significantly higher or lower compared to the average over the neighboring region. With respect to the triaxiality $\eta$, this result indicates that, not surprisingly, fatigue crack nucleation in SGI is not controlled by the same mechanism responsible for the initiation of ductile fracture during monotonic tensile loading. Indeed, it is well known that the latter proceeds via nucleation, growth and coalescence of micro-voids, a process which is enhanced by high values of $\eta$. Rather, the fact that $\Delta \varepsilon_{e q}^{p l}$ and $\varepsilon_{I, \text { max }}^{p l}$ are associated with large, positive values of $q_{c}$ of similar magnitude suggests that crack nucleation is mainly controlled by the plastic strain. Nevertheless, the large values of the standard deviation prevent making a solid conclusion on this point. It can be hypothesized that, as in most metallic materials, crack nucleation is caused by the localization of 
irreversible plastic slip [44], which cannot be captured adequately at the scale of the present model where the matrix is considered isotropic and homogeneous (see Section 3.2).

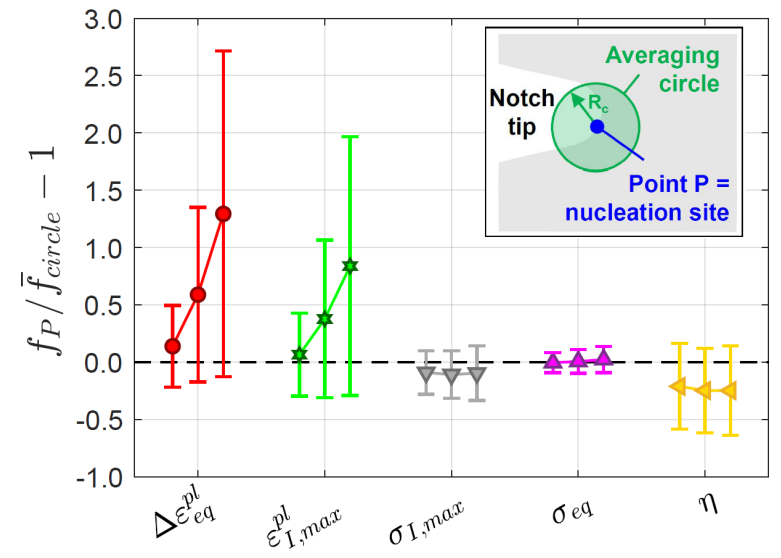

Figure 8 - Quantity $q_{c}=f_{P} / \bar{f}_{\text {circle }}-1$ for the five scalar fields extracted from the notch tip model. For each field, three sets of data are reported, corresponding to (left to right) $R_{c}=20 \mu \mathrm{m}, R_{c}=40 \mu \mathrm{m}$ and $R_{c}=60 \mu \mathrm{m}$. For each $\mathrm{R}_{\mathrm{c}}$-value, the solid marker and the error bars indicate the mean value and the standard deviation, respectively, taken over the $\mathrm{X}-\mathrm{Z}$ slices forming the specimen's thickness.

\subsection{Correlation between micromechanical fields and crack propagation direction}

The results presented in Section 4.1 demonstrate that the crack path in the matrix deviates significantly from the ideal propagation plane. As mentioned in the introduction, this behavior might be connected to the local variations of the micro-mechanical fields caused by the presence of the nodules. To verify this hypothesis, the quantity $q_{a}=f_{Q} / \bar{f}_{\text {arc }}-1$ was evaluated for all the five scalar fields mentioned in the previous section. The difference compared to $q_{c}$ is that $\bar{f}_{a r c}$ is not the average over a circle, but over the arc of radius $\mathrm{R}_{\mathrm{a}}$ and length $2 \mathrm{R}_{\mathrm{a}}$, centered on the crack nucleation site (point $\mathrm{P}$ ) and positioned symmetrically with respect to the point $\mathrm{Q}$ at which the arc intersects the crack (see inset in Figure 9 (a)). The idea 
is that $q_{a}$ should provide a quantitative measure of whether the crack propagated along a direction characterized by values of $f$ higher or lower than the average.

Figure 9 (a) shows $q_{a}$ computed based on the numerical predictions of the notch tip model. The mean values very close to zero indicate that no correlation seems to exist between the crack growth direction and the five scalar fields considered. A possible explanation is that the crack, once nucleated, causes a stress redistribution during its propagation that is not accounted for in the notch tip model. To verify this, the calculation of $q_{a}$ was repeated considering the output of the crack tip model and centering the arc at the crack tip after 40000 cycles (point $\mathrm{P}^{\prime}$ in the inset in Figure 9 (b)). Figure 9 (b) indicates that, again, no correlation can be identified despite the fact that the accurate geometry of the crack is included in this second model.

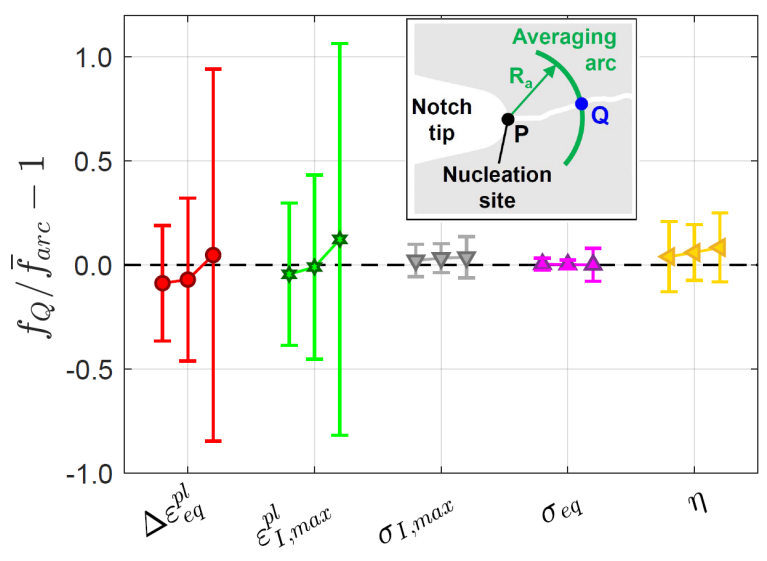

(a)

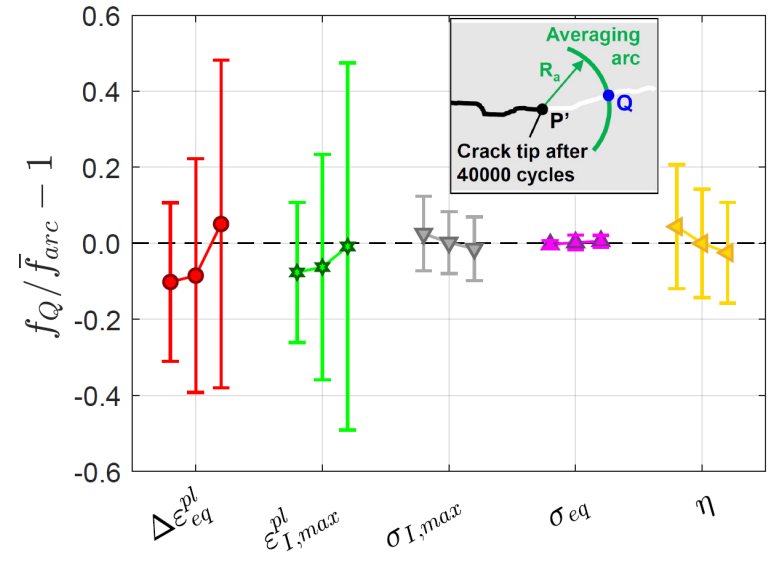

(b)

Figure 9 - Quantity $q_{a}=f_{Q} / \bar{f}_{\text {arc }}-1$ for the five scalar fields extracted from (a) the notch tip model and (b) the crack tip model. For each field, three sets of data are reported, corresponding to (left to right) $R_{a}=20 \mu \mathrm{m}, R_{a}=40$ $\mu \mathrm{m}$ and $\mathrm{R}_{\mathrm{a}}=60 \mu \mathrm{m}$. For each $\mathrm{R}_{\mathrm{a}}$-value, the solid marker and the error bars indicate the mean value and the standard deviation, respectively, taken over the $\mathrm{X}-\mathrm{Z}$ slices forming the specimen's thickness. 
To a certain extent, the absence of a clear correlation with the scalar micro-mechanical fields is not surprising. Indeed, it is well known that in many materials the crack at the macro-scale tends to propagate following the direction perpendicular to the maximum principal stress, which is a vector quantity. To verify if the same holds at the level of the SGI microstructure, the angle $\Delta \theta_{Z X}$ between the predicted crack propagation direction in the X-Z plane - defined as the direction perpendicular to the projection of the maximum principal stress onto the $\mathrm{X}$ $\mathrm{Z}$ plane - and the real propagation direction measured from the reconstructed crack geometry was computed. This operation was performed for all X-Z slices; for each slice, it was repeated five times considering points located at increasing distances $\Delta a$ from either the crack nucleation site (notch tip model) or the crack tip after 40000 cycles (crack tip model). The results are presented in Figure 10 in the form of box plots, where the red horizontal band indicates the median of the $\Delta \theta_{Z X}$ values over the different $X-Z$ slices, the box edges represent the $25^{\text {th }}$ and the $75^{\text {th }}$ percentile and the whiskers extend to cover a length of 2.7 times the standard deviation. It can be seen that, at $\Delta a=0$, the median is close to zero and the absolute value of $\Delta \theta_{Z X}$ is less than $\approx 10$ degrees for $50 \%$ of the $\mathrm{X}-\mathrm{Z}$ slices. This is true for both cases. As $\Delta a$ increases, the dispersion of $\Delta \theta_{Z X}$ in terms of distance between the $25^{\text {th }}$ and the $75^{\text {th }}$ percentile increases, probably because the models do not account for the redistribution of the stress field occurring as the crack propagates. The sensitivity of the results of Figure 10 to critical model parameters like boundary conditions and matrix plastic hardening was also checked - see Appendix A and B - indicating minimal variations. Therefore, the present analysis supports the hypothesis that the crack propagation path in the SGI matrix is determined by the micro-mechanical fields and specifically by the maximum principal stress 
direction, which in turn is significantly influenced by the presence of the nodules. In this respect, it is worth remarking that this result was found by modeling the matrix as homogeneous and isotropic, meaning that the impact of grain-related effects is likely to be subordinate to that of the nodules in this particular case.

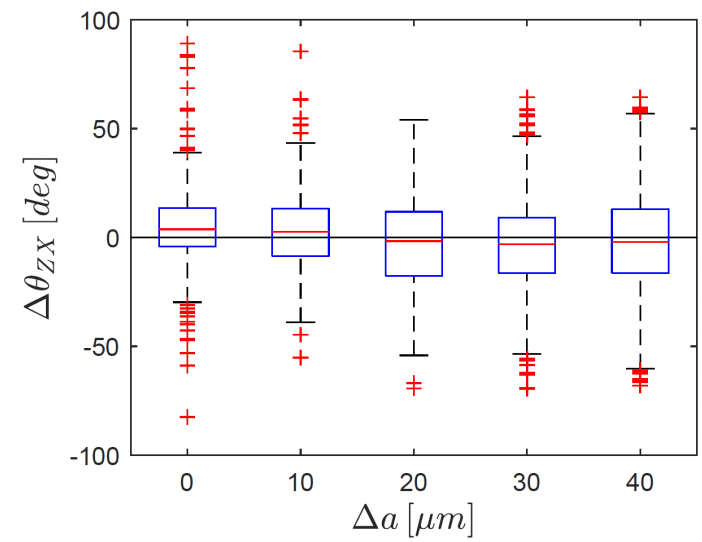

(a)

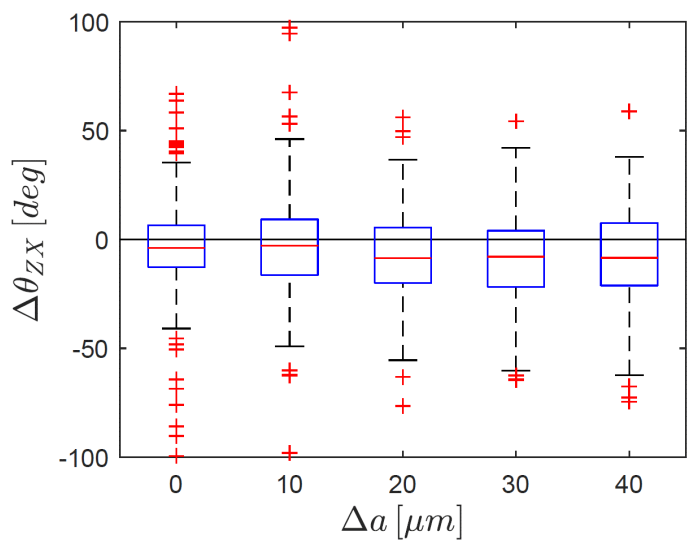

(b)

Figure 10 - Statistical box plots of the angle $\Delta \theta_{Z X}$ between the predicted and the measured crack propagation directions in the $\mathrm{X}-\mathrm{Z}$ plane. (a) Notch tip model. (b) Crack tip model. $\Delta a$ is the distance from either the crack nucleation site (notch tip model) or the crack tip after 40000 cycles (crack tip model).

\section{Crack step formation mechanism}

The crack geometry reconstructed with the technique reported in Section 2.3 allows a detailed examination of the distribution of the nodules around the step-like features of the crack surface, which, as already mentioned, have been associated in the literature with a reduction in the crack growth rate. In this respect, a careful inspection of Figure 7 (b) and (c) reveals that the corresponding steps seem to originate from the large nodules marked with the red arrows. The qualitative impression is that the crack front is initially straight and splits up after encountering such large nodules, hence generating a step with step wall parallel to the crack 
growth direction. This observation, combined with the fact that the nodules act as crack attractors [12,15], allows formulating a hypothesis for the mechanisms of formation of these steps, which is schematized in Figure 11 (a). The idea is to consider a straight horizontal crack front that moves along the Z-axis (i) and, at a certain point, reaches a nodule (ii). This causes debonding between the nodule and the matrix, so that the crack front is now composed of two halves, separated by a cavity. As cyclic loading continues, the two front halves grow independently around the nodule. During this stage, it can happen that the distribution of the neighboring nodules is such that the front halves are attracted one upwards and the other downwards (iii). In such a case, it might be that the two halves are not able to merge anymore after passing the nodule, as now they propagate on planes located at different heights (iv). Therefore, in order for the fracture process to continue, the vertical ligament between the two halves of the crack front must break up, leading to the formation of the step (v). Clearly, this process would require extra energy and could explain the reduction in the crack growth rate detected experimentally.

The critical assumption of the present hypothesis is the existence of an asymmetric driving force such that the two halves of the crack front start propagating in different directions while growing around the nodule. According to the outcome of Section 4.3, the maximum principal stress determines the crack growth direction. Therefore, the proposed mechanism implies that the micro-mechanical stress fields must have "pulled" the crack in different directions on the opposite sides of the nodules marked with the red arrows in Figure 7 (b) and (c). To verify this, an additional finite element model of the microstructure near the region visualized in Figure 7 (b) was developed following the procedure described in Section 3. For 
convenience, the crack front was assumed to follow the red line depicted in the same figure, which intersects the nodule marked with the red arrow just before the crack starts splitting to generate the step. The model was used to compute the expected crack propagation angle $\theta_{Z X}$ along the crack front based on the direction perpendicular to the projection of the maximum principal stress onto the X-Z plane. The result is reported in Figure 11 (b) and shows that the values of $\theta_{Z X}$ differ significantly between the two sides of the nodule indeed, hence confirming the existence of an asymmetric micro-mechanical driving force consistent with the hypothesized mechanism of step formation. Furthermore, it should be noted that, despite the strong assumptions made in the model, a relatively good qualitative agreement between the measured and the predicted crack path around the nodule is observed.

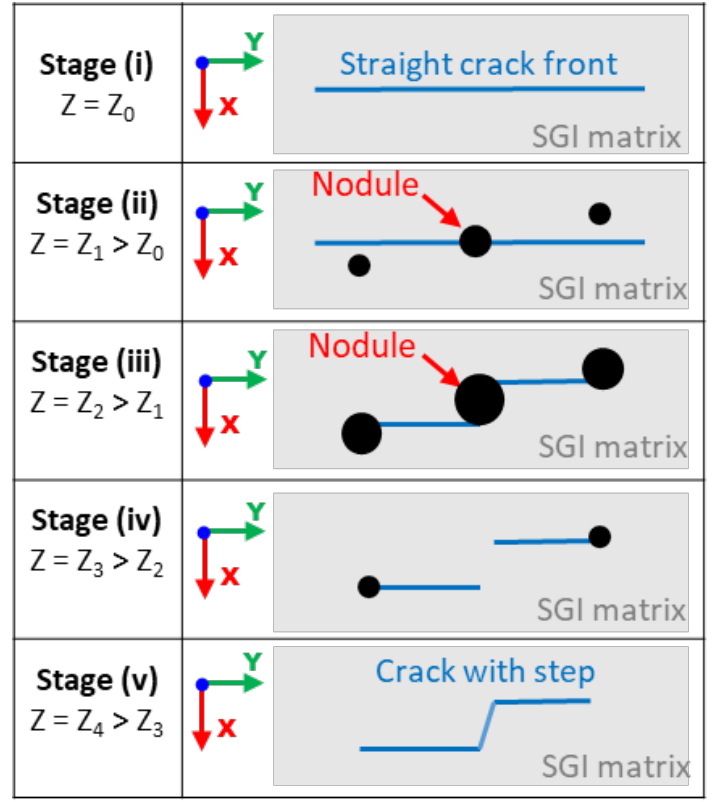

(a)

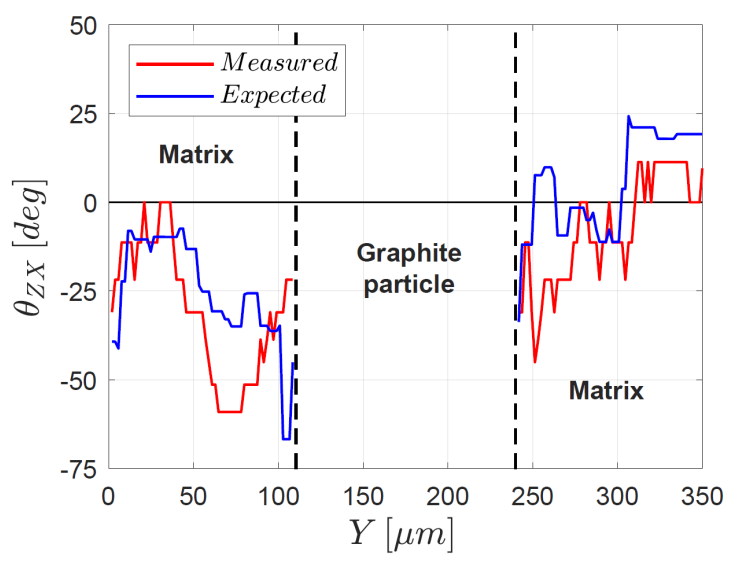

(b)

Figure 11 - (a) Hypothesized mechanism of formation of the steps detected on the fatigue crack surface. (b) Comparison between the measured crack propagation angle along the red continuous line of Figure 7 (b) and that expected based on the maximum principal stress direction predicted by the finite element model. 


\section{Conclusions}

The accurate $3 \mathrm{D}$ geometry of the fatigue crack propagating in a compact tension specimen made of SGI was reconstructed by means of X-ray tomography combined with a dedicated segmentation procedure. The crack nucleation site and the crack propagation direction in the SGI matrix were compared to the micro-mechanical fields predicted by microstructureresolved finite element models with boundary conditions measured in-situ via digital image correlation. In the models, the matrix was assumed homogeneous and isotropic elastoplastic with combined isotropic and kinematic hardening, whereas the nodules were modelled as anisotropic linear elastic with inhomogeneous elastic moduli. Matrix-nodule debonding was also included in the models. The main conclusions can be summarized as follows:

- The proposed segmentation procedure allowed showing that the crack deviations from the ideal propagation plane are a few times larger than the average size of the nodules. This demonstrates that the crack roughness cannot be considered the result of a planar crack whose surface merely "adapts" to the shape of the nodules intercepted along the way.

- No relationship was found between the crack nucleation site and the stress-related micro-mechanical fields. Only a weak correlation with the plastic strain was detected, suggesting that the fatigue crack nucleation is controlled by plastic phenomena that cannot be captured adequately at the scale of the present models.

- A clear correlation was found between the crack growth direction and the direction perpendicular to the maximum principal stress. As the matrix grain structure was 
neglected in the models, this suggests that the crack propagation path could be predicted well via micro-mechanical calculations accounting for the accurate graphite topology and loading conditions.

- The formation of the distinct step-like features of the crack was proposed to be the result of an asymmetric micro-mechanical driving force acting on the crack around the nodules. The presence of such driving force was verified for the largest of the steps detected in the present work.

\section{Acknowledgements}

This work was carried out within the frame of the project "Optimised high performance ductile cast iron using 4D X-ray analysis and microstructural modelling”, Danish Research Council for Independent Research, grant no. 8022-00085B. The authors gratefully acknowledge: Dr. Karl Martin Pedersen and Dr. Sanita Zike from Siemens Gamesa Renewable Energy A/S, for providing the material used in the investigation and performing the tensile monotonic and cyclic tests; Dr. Chaoling Xu and M.Sc. Steffen Rasmussen from Technical University of Denmark, for providing support during the preparation of the specimen and the crack growth test; Ass. Prof. Johan Hoefnagels and M.Sc. Aslan Mohammadpourshoorbakhlou, from Eindhoven University of Technology, for help with the DIC analysis and the meshing toolbox.

\section{Appendix A: Matrix plastic properties}

The elastoplastic model describing the constitutive behavior of the matrix was calibrated by constructing a Representative Volume Element (RVE) of the material at hand and, assuming 
the nodules' properties known, subsequently tuning the matrix plastic parameters such that the macroscopic response of the RVE matches the cyclic behavior of SGI, shown in Figure 1 (b). In the following, the details of this procedure are reported.

First, a suitable RVE was identified in the region of CT scan \#1 located ahead of the notch tip. Figure A1 (a) shows the graphite volume fraction $f_{g}$ and the number of nodules $N_{p}$ in the RVE as functions of the selected RVE size. It can be seen that a stabilization in $f_{g}$ is only reached at $\approx 900 \mu \mathrm{m}$. As $f_{g}$ is known to have a strong impact on the mechanical properties of SGI, it follows that the size of the RVE cannot be less than this value. On the other hand, performing material properties identification involving many repetitive computations with an $\mathrm{RVE}$ containing $\approx 200$ nodules would be computationally prohibitive. Therefore, a smaller RVE of size $300 \mu \mathrm{m}$ containing 8 nodules was selected, where, however, the nodules were expanded isotropically about their centroids by $\approx 4 \%$ in volume in order to achieve the desired $f_{g}$ value of $13.9 \%$.

The RVE was next meshed following the procedure outlined in Section 3.1 - see the mesh in Figure A1 (b) - and the properties of the microstructural constituents assigned according to Section 3.2. The plastic properties reported in [39] were initially used for the matrix. Periodic boundary conditions were imposed on the non-periodic RVE mesh following the approach of Nguyen et al. [45], using Lagrange polynomials of degree 9. The macroscopic stress was determined as the average of the corresponding microscopic quantity, computed with the simplified formula described in [46].

First, a mesh sensitivity analysis was carried out. Sub-diving each element of the mesh shown in Figure A1 (b) into eight sub-elements changed the predicted macroscopic yield stress by 
$0.2 \%$ only. The local microscopic von Mises stress in two matrix points close to a nodule and far from it changed by $0.3 \%$ and $1.2 \%$, respectively. Therefore, the mesh size was considered appropriate (for this reason, the same meshing parameters were used to generate the meshes of the two models described in Section 3.1). After this, tensile loading was simulated along different directions in order to check the level of isotropy of the RVE, showing almost no differences. Finally, the size of the RVE was tested by comparing its response to that of a larger RVE of size $400 \mu \mathrm{m}$, again resulting in negligible variations.

Having verified the quality of the RVE, the calibration of the plastic parameters of the matrix was carried out by means of a conjugate gradient algorithm. First, $H$ and $\gamma$ were determined by fitting the data of Figure 1 (b) limited to the last cycle, assuming that negligible isotropic hardening takes place within that cycle. Then, the values of $Q_{0}, Q_{\infty}$ and $b$ were estimated by fitting the full cyclic data. The identified parameter values are given in Table 2 .

In order to check the impact of the identification procedure on the main findings of the present investigation, the sensitivity of the results of Figure 8, Figure 9 and Figure 10 to the matrix hardening parameters was assessed. To this end, the corresponding finite element simulations were repeated neglecting the kinematic part of the hardening in the constitutive model of the matrix. Hardly any changes in the results were detected. This can be explained partly by the positive fatigue ratio applied to the compact tension specimen during cyclic loading, and partly by the relatively low amount of hardening exhibited by the matrix of the SGI at hand. 

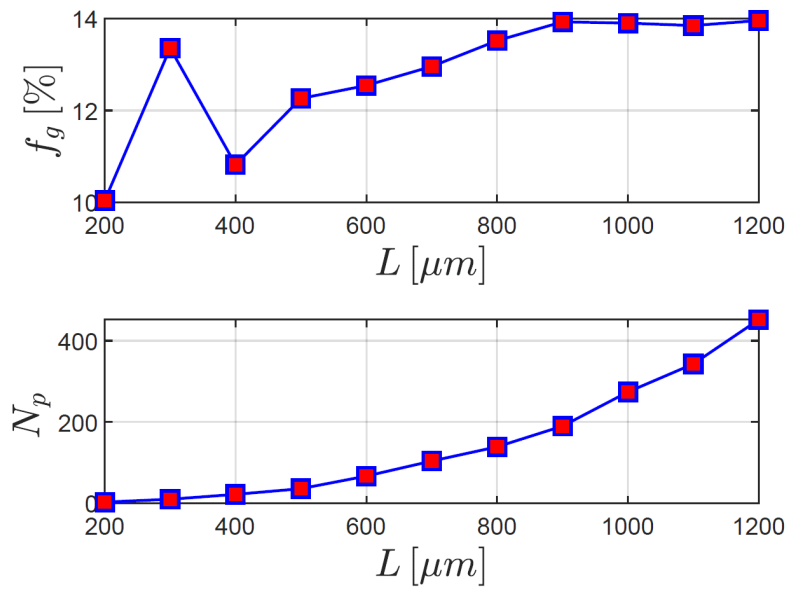

(a)

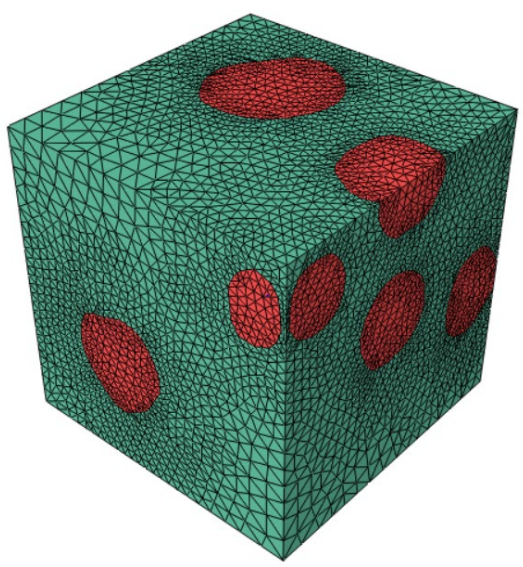

(b)

Figure A1 - (a) Graphite volume fraction and number of nodules in the RVE as functions of the RVE size $L$.

(b) Mesh of the RVE used for calibrating the matrix plastic parameters (red = nodules, green $=$ matrix).

\section{Appendix B: Displacement boundary conditions}

The DIC displacement components at maximum load prescribed along the boundaries of both the notch tip and crack tip models are shown in Figure B1 (the values are extracted from the contours of Figure 6). For comparison, in the same figure the "ideal" displacement components estimated with a 2D finite element model of the compact tension specimen that assumes homogenized material behavior are reported. It can be seen that some deviations between the two data sets exist, which seem to be more pronounced in the case of the crack tip model, i.e. after 40000 loading cycles. This fact is not surprising, as recent studies have emphasized that the boundary conditions measured in-situ can differ substantially from the “ideal" values estimated with simplified models, see e.g. [47].

The extent to which the choice of the boundary condition affects the outcome of the present investigation was evaluated by re-computing Figure 8, Figure 9 and Figure 10 based on 
simulations where the "ideal" displacements were prescribed along the model boundaries instead of those measured via DIC. Negligible differences were found, indicating that the corresponding results are, to a certain degree, insensitive to the specific values of the boundary conditions. A possible explanation is that the size of the models - see Figure 5 - is sufficiently large to minimize the effect of small fluctuations in the boundary conditions on the pattern of the micro-mechanical fields in the region of interest, i.e. in front of the notch/crack tip. This hypothesis is qualitatively confirmed in Figure B2, which compares the contours of the maximum principal stress on one section of the crack tip model, computed using the two sets of displacement data as boundary conditions.
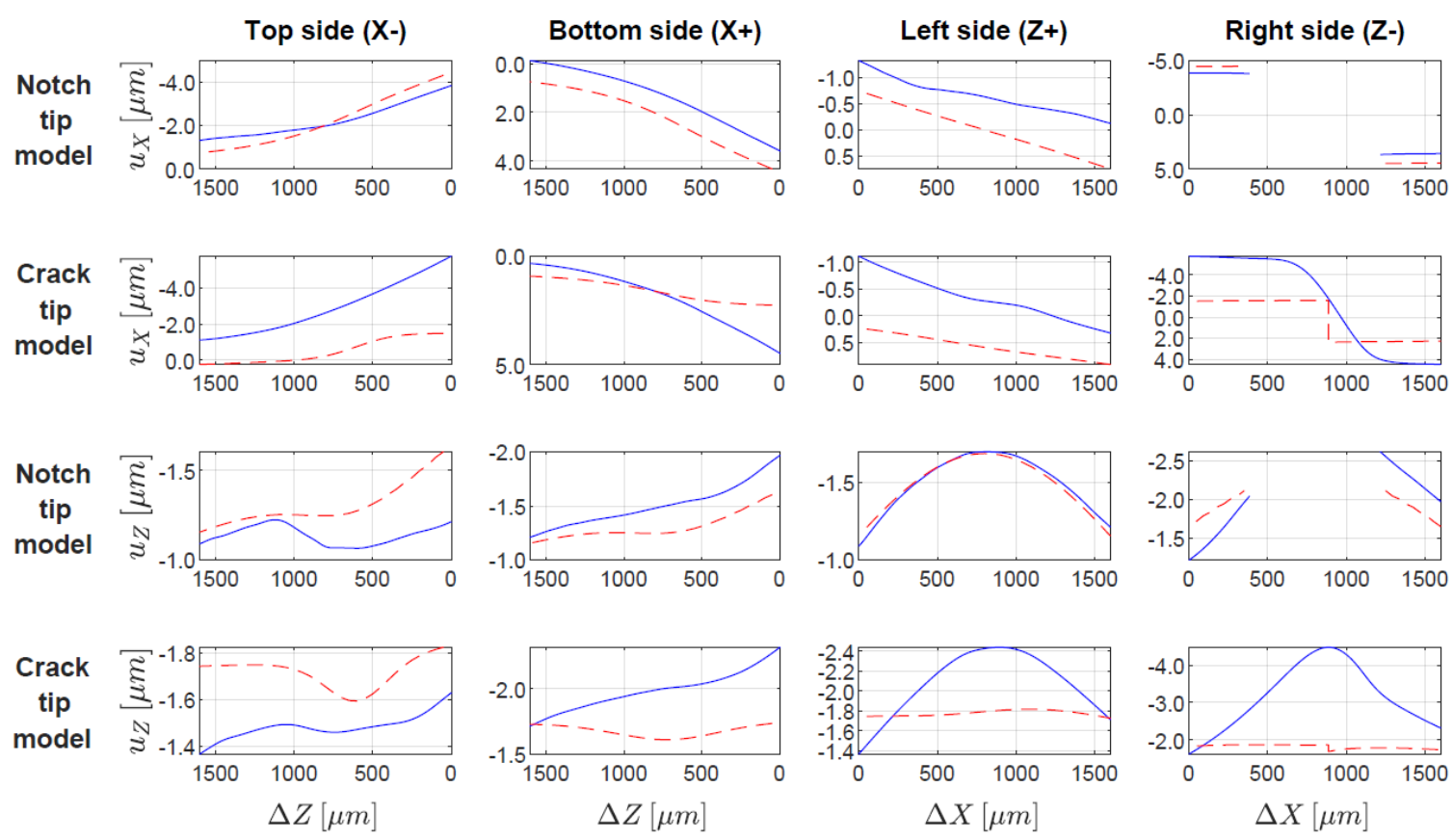

Figure B1 - Displacement at maximum load prescribed along the model boundaries either reconstructed using DIC (blue continuous line) or estimated with a 2D finite element simulation of the homogeneous compact tension specimen (red dashed line). Values are in $\mu \mathrm{m}$. 


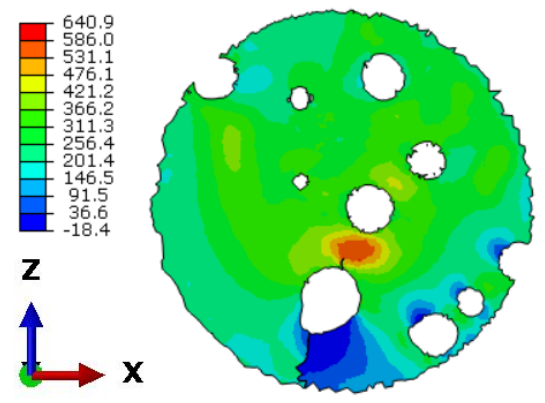

(a)

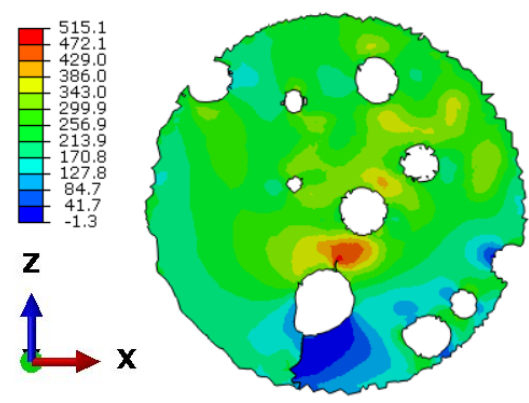

(b)

Figure B2 - Contour of the maximum principal stress in the matrix region at peak load in MPa. The data relates to a single $\mathrm{X}-\mathrm{Z}$ section of the crack tip model. (a) Measured boundary conditions from DIC. (b) Ideal boundary conditions estimated with a 2D finite element simulation of the homogeneous compact tension specimen.

\section{References}

[1] D.M. Stefanescu, ed., ASM Handbook, Cast Iron Science and Technology, Volume 1 A, ASM International, 2017.

[2] P. Čanžar, Z. Tonković, J. Kodvanj, Microstructure influence on fatigue behaviour of nodular cast iron, Mater. Sci. Eng. A. 556 (2012) 88-99. doi:10.1016/j.msea.2012.06.062.

[3] E. Foglio, D. Lusuardi, A. Pola, G.M. La Vecchia, M. Gelfi, Fatigue design of heavy section ductile irons: Influence of chunky graphite, Mater. Des. 111 (2016) 353-361. doi:10.1016/j.matdes.2016.09.002.

[4] K. Mukherjee, S. Fæster, N. Hansen, A.B. Dahl, C. Gundlach, J.O. Frandsen, A. Sturlason, Graphite nodules in fatigue-tested cast iron characterized in 2D and 3D, Mater. Charact. 129 (2017) 169-178. doi:10.1016/j.matchar.2017.04.024.

[5] D.M. Stefanescu, G. Alonso, P. Larrañaga, E. De La Fuente, R. Suarez, On the crystallization of graphite from liquid iron-carbon-silicon melts, Acta Mater. 107 (2016) 102-126. doi:10.1016/j.actamat.2016.01.047.

[6] K. Tokaji, T. Ogawa, K. Shamoto, Fatigue crack propagation in spheroidal- graphite cast irons with different microstructures, 16 (1994) 344-350.

[7] M. Benedetti, V. Fontanari, D. Lusuardi, Effect of graphite morphology on the fatigue and fracture resistance of ferritic ductile cast iron, Eng. Fract. Mech. 206 (2019) 427-441. doi:10.1016/j.engfracmech.2018.12.019.

[8] L. Heine, A. Bezold, C. Broeckmann, Long crack growth and crack closure in high strength nodular cast iron, Eng. Fract. Mech. 192 (2018) 24-53. doi:10.1016/j.engfracmech.2018.02.003.

[9] P. Hubner, H. Schlosser, G. Pusch, H. Biermann, Load history effects in ductile cast iron for wind turbine components, Int. J. Fatigue. 29 (2007) 1788-1796. doi:10.1016/j.ijfatigue.2007.01.012.

[10] G. Hütter, L. Zybell, M. Kuna, Micromechanisms of fracture in nodular cast iron: From experimental findings towards modeling strategies - A review, 144 (2015) 118-141. doi:10.1016/j.engfracmech.2015.06.042.

[11] D.O. Fernandino, R. Boeri, Study of the fracture of ferritic ductile cast iron under different loading conditions, Fatigue Fract. Eng. Mater. Struct. 38 (2015) 610-620. doi:10.1111/ffe.12266. 
[12] F. Iacoviello, V. Di Cocco, C. Bellini, Fatigue crack propagation and damaging micromechanisms in Ductile Cast Irons, Int. J. Fatigue. 124 (2019) 48-54. doi:10.1016/j.ijfatigue.2019.02.030.

[13] J. Hosdez, N. Limodin, D. Najjar, J.-F. Witz, E. Charkaluk, P. Osmond, A. Forré, F. Szmytka, Fatigue crack growth in compacted and spheroidal graphite cast irons, Int. J. Fatigue. (2019) 105319. doi:https://doi.org/10.1016/j.jpatigue.2019.105319.

[14] C. Verdu, J. Adrien, J.Y. Buffière, Three-dimensional shape of the early stages of fatigue cracks nucleated in nodular cast iron, Mater. Sci. Eng. A. 483-484 (2008) 402-405. doi:10.1016/j.msea.2006.09.178.

[15] S. Sujakhu, S. Castagne, M. Sakaguchi, K.A. Kasvayee, E. Ghassemali, A.E.W. Jarfors, W. Wang, On the fatigue damage micromechanisms in Si-solution-strengthened spheroidal graphite cast iron, Fatigue Fract. Eng. Mater. Struct. 41 (2018) 625-641. doi:10.1111/ffe.12723.

[16] A. Ayyar, N. Chawla, Microstructure-based modeling of crack growth in particle reinforced composites, Compos. Sci. Technol. 66 (2006) 1980-1994. doi:10.1016/j.compscitech.2006.01.007.

[17] A. Ayyar, N. Chawla, Microstructure-based modeling of the influence of particle spatial distribution and fracture on crack growth in particle-reinforced composites, Acta Mater. 55 (2007) 6064-6073. doi:10.1016/j.actamat.2007.06.044.

[18] Y.X. Gao, J.Z. Yi, P.D. Lee, T.C. Lindley, A micro-cell model of the effect of microstructure and defects on fatigue resistance in cast aluminum alloys, Acta Mater. 52 (2004) 5435-5449. doi:10.1016/j.actamat.2004.07.035.

[19] J.Z. Yi, Y.X. Gao, P.D. Lee, T.C. Lindley, Effect of Fe-content on fatigue crack initiation and propagation in a cast aluminum-silicon alloy (A356-T6), Mater. Sci. Eng. A. 386 (2004) 396-407. doi:10.1016/j.msea.2004.07.044.

[20] K.A. Kasvayee, E. Ghassemali, K. Salomonsson, S. Sujakhu, S. Castagne, A.E.W. Jarfors, Microstructural strain mapping during in-situ cyclic testing of ductile iron, Mater. Charact. 140 (2018) 333-339. doi:10.1016/j.matchar.2018.04.017.

[21] T. Wigger, C. Lupton, S. Alshammrei, J. Tong, T.J. Marrow, P. Earp, M.L. Zhu, D.Q. Wang, T. Connolley, In situ mapping of normal strains in the field of a growing fatigue crack in a steel weld using digital image correlation and energy dispersive synchrotron X-ray diffraction, Int. J. Fatigue. 115 (2018) 11-19. doi:10.1016/j.ijfatigue.2018.05.016.

[22] Y.B. Zhang, T. Andriollo, S. Fæster, R. Barabash, R. Xu, N. Tiedje, J. Thorborg, J. Hattel, D. Juul Jensen, N. Hansen, Microstructure and residual elastic strain at graphite nodules in ductile cast iron analyzed by synchrotron X-ray microdiffraction, Acta Mater. 167 (2019) 221-230. doi:10.1016/j.actamat.2019.01.038.

[23] Y.B. Zhang, T. Andriollo, S. Fæster, W. Liu, J. Hattel, R.I. Barabash, Three-dimensional local residual stress and orientation gradients near graphite nodules in ductile cast iron, Acta Mater. 121 (2016) 173180. doi:10.1016/j.actamat.2016.09.009.

[24] N. Limodin, J. Réthoré, J.Y. Buffière, A. Gravouil, F. Hild, S. Roux, Crack closure and stress intensity factor measurements in nodular graphite cast iron using three-dimensional correlation of laboratory Xray microtomography images, Acta Mater. 57 (2009) 4090-4101. doi:10.1016/j.actamat.2009.05.005.

[25] N. Limodin, J. Réthoré, J.Y. Buffière, F. Hild, S. Roux, W. Ludwig, J. Rannou, A. Gravouil, Influence of closure on the 3D propagation of fatigue cracks in a nodular cast iron investigated by X-ray tomography and 3D volume correlation, Acta Mater. 58 (2010) 2957-2967. doi:10.1016/j.actamat.2010.01.024.

[26] J. Lachambre, J. Réthoré, A. Weck, J.Y. Buffiere, Extraction of stress intensity factors for 3D small fatigue cracks using digital volume correlation and X-ray tomography, Int. J. Fatigue. 71 (2015) 3-10. doi:10.1016/j.ijfatigue.2014.03.022.

[27] ASTM International, Standard E647 - 15: Standard Test Method for Measurement of Fatigue Crack Growth Rates, (2018).

[28] L.A. Feldkamp, L.. Davis, J.W. Kress, Practical cone-beam algorithm, J. Opt. Soc. Am. A. 1 (1984) 612-619.

[29] A. Buljac, C. Jailin, A. Mendoza, J. Neggers, T. Taillandier-Thomas, A. Bouterf, B. Smaniotto, F. Hild, S. Roux, Digital Volume Correlation: Review of Progress and Challenges, Exp. Mech. 58 (2018) 661708. doi:10.1007/s11340-018-0390-7. 
[30] J. Rannou, N. Limodin, J. Réthoré, A. Gravouil, W. Ludwig, M.C. Baïetto-Dubourg, J.Y. Buffière, A. Combescure, F. Hild, S. Roux, Three dimensional experimental and numerical multiscale analysis of a fatigue crack, Comput. Methods Appl. Mech. Eng. 199 (2010) 1307-1325. doi:10.1016/j.cma.2009.09.013.

[31] Thermo Scientific ${ }^{\mathrm{TM}}$, Avizo 9.6, (2019). www.thermofisher.com.

[32] A. Jain, Fundamentals of Digital Image Processing, Prentice-Hall, 1989.

[33] K.M. Pedersen, N.S. Tiedje, Graphite nodule count and size distribution in thin- walled ductile cast iron, Mater. Charact. 59 (2008) 1111-1121.

[34] K. Ehab Moustafa Kamel, B. Sonon, T.J. Massart, An integrated approach for the conformal discretization of complex inclusion-based microstructures, Comput. Mech. (2019). doi:10.1007/s00466-019-01693-4.

[35] T. Andriollo, Y.B. Zhang, S. Fæster, J. Thorborg, J. Hattel, Impact of micro-scale residual stress on insitu tensile testing of ductile cast iron: Digital volume correlation vs. model with fully resolved microstructure vs. periodic unit cell, J. Mech. Phys. Solids. 125 (2019) 714-735. doi:10.1016/J.JMPS.2019.01.021.

[36] C. Bellini, V. Di Cocco, G. Favaro, F. Iacoviello, L. Sorrentino, Ductile cast irons: Microstructure influence on the fatigue initiation mechanisms, Fatigue Fract. Eng. Mater. Struct. (2019) 1-11. doi:10.1111/ffe. 13100 .

[37] T. Andriollo, S. Fæster, G. Winther, Probing the structure and mechanical properties of the graphite nodules in ductile cast irons via nano-indentation, Mech. Mater. 122 (2018) 85-95. doi:10.1016/j.mechmat.2018.03.010.

[38] Dassault Systèmes Simulia Corp., Abaqus 3DEXPERIENCE R2017x, Analysis User's Guide section 37.1.10, (2017).

[39] F. Rabold, M. Kuna, Cell model simulation of void growth in nodular cast iron under cyclic loading, Comput. Mater. Sci. 32 (2005) 489-497. doi:10.1016/j.commatsci.2004.09.016.

[40] T. Andriollo, K. Hellström, M.R. Sonne, J. Thorborg, N. Tiedje, J. Hattel, Uncovering the local inelastic interactions during manufacture of ductile cast iron: How the substructure of the graphite particles can induce residual stress concentrations in the matrix, J. Mech. Phys. Solids. 111 (2018) 333-357. doi:10.1016/j.jmps.2017.11.005.

[41] K. Matouš, M.G.D. Geers, V.G. Kouznetsova, A. Gillman, A review of predictive nonlinear theories for multiscale modeling of heterogeneous materials, J. Comput. Phys. 330 (2017) 192-220. doi:10.1016/j.jcp.2016.10.070.

[42] G. Fischer, J. Nellesen, N.B. Anar, K. Ehrig, H. Riesemeier, W. Tillmann, 3D analysis of microdeformation in VHCF-loaded nodular cast iron by $\mu$ CT, Mater. Sci. Eng. A. 577 (2013) 202-209. doi:10.1016/j.msea.2013.04.057.

[43] T. Mottitschka, G. Pusch, H. Biermann, L. Zybell, M. Kuna, Influence of graphite spherical size on fatigue behaviour and fracture toughness of ductile cast iron EN-GJS-400-18LT, Int. J. Mater. Res. 103 (2012) 87-96. doi:10.3139/146.110636.

[44] F.P.E. Dunne, Fatigue crack nucleation: Mechanistic modelling across the length scales, Curr. Opin. Solid State Mater. Sci. 18 (2014) 170-179. doi:10.1016/j.cossms.2014.02.005.

[45] V.-D. Nguyen, E. Béchet, C. Geuzaine, L. Noels, Imposing periodic boundary condition on arbitrary meshes by polynomial interpolation, Comput. Mater. Sci. 55 (2012) 390-406. doi:10.1016/j.commatsci.2011.10.017.

[46] A.I. Akpoyomare, M.I. Okereke, M.S. Bingley, Virtual testing of composites : Imposing periodic boundary conditions on general finite element meshes, Compos. Struct. 160 (2017) 983-994. doi:10.1016/j.compstruct.2016.10.114.

[47] M. Shakoor, A. Buljac, J. Neggers, F. Hild, T.F. Morgeneyer, L. Helfen, M. Bernacki, P.-O. Bouchard, On the choice of boundary conditions for micromechanical simulations based on 3D imaging, Int. J. Solids Struct. 112 (2017) 83-96. doi:https://doi.org/10.1016/j.ijsolstr.2017.02.018. 